\title{
Instrumento BALORA para la valoración de la gravedad de las situaciones de riesgo y desamparo infantil: elaboración, implantación, fundamentos conceptuales y contenido
}

\section{Ignacia Arruabarrena Madariaga}

Facultad de Psicología, Universidad del País Vasco/Euskal Herriko Unibertsitate (UPV/EHU)

ignacia.arruabarrena@ehu.eus

\section{Miguel Ángel Hurtado Pedroso}

Dirección de Política Familiar y Diversidad, Gobierno Vasco

ma-hurtado@euskadi.eus

BALORA tresna 3/2005 Legea, otsailaren 18koa, Haurrak eta Nerabeak zaintzeko eta babestekoa, zeinak Eusko Jaurlaritzari, foru-aldundiei eta udaletxeei arrisku- eta babesgabetasun-egoeren larritasuna ebaluatzeko tresna tekniko baten lanketa eta onarpena eskatzen baitzien. Artikulu honetan tresna horren lanketa-prozesua azaltzen da, haren onarpena 2011ko azaroan, autonomia-erkidegoan orokortu den ezarpena, eta 2017ko maiatzean eguneratu eta onartutako dekretu berria. Haren eragina neurtzeko ikerketetako emaitzak azaltzen dira, haren oinarri kontzeptualak, irizpide teknikoak eta edukia deskribatu, eta erabilera zuzena egiteko ohar batzuk nabarmentzen dira.

\section{GAKO-HITZAK:}

Haur-babesgabetasuna, arrisku-egoera, babesgabetasun-egoera, ebaluazioa.
El instrumento BALORA se diseñó con el objetivo de responder al mandato de la Ley $3 / 2005$, de 18 de febrero, de Atención y Protección a la Infancia y la Adolescencia, que encomendaba al Gobierno Vasco, diputaciones forales y ayuntamientos la elaboración y aprobación de un instrumento técnico para la valoración de la gravedad de las situaciones de riesgo y desamparo. Este artículo describe el proceso llevado a cabo para la elaboración de dicho instrumento, su aprobación como decreto en noviembre 2011, su implantación generalizada en la comunidad autónoma, y su revisión, actualización y aprobación en un nuevo decreto en mayo 2017. Se presentan los resultados de los estudios realizados para analizar su impacto, se describen sus fundamentos conceptuales, criterios técnicos, contenido, y se apuntan algunas indicaciones para su correcta utilización.

\section{Palabras Clave:}

Desprotección infantil, situación de riesgo, situación de desamparo, valoración. 


\section{Introducción}

El Instrumento para la valoración de la gravedad de las situaciones de riesgo y desamparo en los Servicios Sociales Municipales y Territoriales ${ }^{1}$ de Atención y Protección a la Infancia y Adolescencia en la Comunidad Autónoma del País Vasco (BALORA) se diseñó con el objetivo de responder a las disposiciones establecidas en los artículos 49, 53 y 54 de la Ley $3 / 2005$, de 18 de febrero, de Atención y Protección a la Infancia y la Adolescencia (en adelante Ley 3/2005) con relación a la investigación y valoración de las situaciones de riesgo y desamparo. En dichos artículos se indicaba, entre otras cuestiones, que la valoración de la gravedad de estas situaciones debía hacerse mediante la aplicación "de instrumentos técnicos validados al efecto", encomendando a Gobierno Vasco, diputaciones forales y ayuntamientos la elaboración y aprobación de un instrumento básico con ese objetivo. Dicho instrumento revestía gran importancia para la distribución de competencias entre servicios sociales municipales y de los distintos territorios, pues era la gravedad el criterio utilizado por la ley para determinar qué situaciones correspondía atender a cada uno de ellos: riesgo leve y moderado a los primeros, y riesgo grave y desamparo a los segundos. Este artículo describe el proceso llevado a cabo para la elaboración e implantación de dicho instrumento (en adelante, BALORA), así como sus fundamentos conceptuales y contenido.

\section{Proceso de elaboración e implantación}

Conocer el proceso de elaboración e implantación del instrumento BALORA no es un ejercicio secundario. Por un parte, conocer cómo se diseñó permite constatar que dispone de una base teórica y técnica sólida, de evidencia cuantitativa y cualitativa de su validez, y que es fruto de un proceso de trabajo altamente participativo. Por otra parte, conocer cómo se llevó a cabo su implantación muestra que no se limitó a su mera difusión o a la provisión de formación sobre su uso. Si se hubiera hecho así, es probable que hubiera sucedido lo mismo que con otros instrumentos técnicos, manuales de actuación o nuevos procedimientos de intervención que han quedado en el cajón o desvirtuados e irreconocibles en su aplicación en la práctica profesional, incapaces de abrirse paso en un ámbito de trabajo mayoritariamente sobrecargado en el que resulta difícil para profesionales y servicios disponer del tiempo y energía requeridos para incorporar y adaptarse a cambios en su quehacer profesional. Para ser exitoso, el proceso de implantación de cualquier nueva metodología de trabajo que implique a un número importante de profesionales y servicios en los que, además, hay mucha movilidad profesional requiere de la provisión de apoyo técnico

${ }^{1}$ En el contexto de este artículo, el término territorial se refiere a los territorios históricos de Gipuzkoa, Bizkaia y Álava. y monitorización continuadas, sobre todo en las primeras fases del proceso. Así se hizo en el caso del instrumento BALORA, aunque es muy posible que aun con esas actuaciones no se hubiera conseguido su implantación generalizada si finalmente no se hubiera procedido a su aprobación como decreto, es decir, a la determinación de su uso obligado en los servicios de atención y protección a la infancia y adolescencia de la comunidad autónoma.

En la elaboración e implantación del instrumento BALORA se distinguen dos fases. La primera de ellas, desarrollada entre 2007-2011, culminó con la aprobación del Decreto 230/2011, que contenía la primera versión del instrumento. La segunda fase, desarrollada entre 2014-2017, conllevó su actualización y aprobación en un nuevo decreto (Decreto 152/2017). Ambos procesos fueron promovidos y liderados por el Gobierno Vasco, y contaron con la implicación y participación activas de un amplio número de profesionales y equipos técnicos de los servicios sociales municipales y territoriales de atención y protección a la infancia y adolescencia (incluyendo Eudel, en su calidad de asociación de municipios más representativa a nivel de la Comunidad Autónoma del País Vasco). El núcleo central y garante de continuidad del proceso fue una comisión técnica formada por entre once y catorce profesionales en representación del Gobierno Vasco, las diputaciones forales de Álava, Bizkaia y Gipuzkoa, los ayuntamientos de Vitoria-Gasteiz, Bilbao y Donostia-San Sebastián, y Eudel. La coordinación técnica del trabajo fue llevada a cabo por un equipo de la Asociación Dartington-i para el estudio y la formación en protección infantil y la Facultad de Psicología de la Universidad del País Vasco (UPV/ EHU). El proyecto contó con asesoramiento y supervisión jurídica permanentes.

\subsection{Primera fase: elaboración y difusión de la primera versión del instrumento BALORA (2007-2011)}

La elaboración del instrumento BALORA se inició con la asunción de que cualquier nueva herramienta que se diseñara para ser utilizada en los servicios de atención y protección a la infancia y adolescencia debía ser aplicable en el marco y procedimiento de actuación de dichos servicios y tomar en consideración los instrumentos técnicos que ya se estaban utilizando. En el caso de la Comunidad Autónoma del País Vasco, estos servicios funcionaban de acuerdo a un marco legislativo común, pero mantenían -y siguen manteniendo- un cierto grado de autonomía tanto a nivel territorial como municipal en su esquema organizativo, procedimiento de actuación y gran parte de los instrumentos de trabajo que aplicaban.

Cada territorio histórico disponía de sus respectivos manuales técnicos. En el caso de Gipuzkoa y Álava, los manuales eran de aplicación tanto en servicios sociales municipales como territoriales. El manual disponible en Bizkaia, por el contrario, era de uso 
exclusivo en los servicios sociales territoriales (Diputación Foral de Álava, 2004; Diputación Foral de Bizkaia, 2005; Diputación Foral de Gipuzkoa, 2003). Basándose en el marco legal vigente, los manuales definían de forma algo más precisa las situaciones de riesgo y desamparo, así como el concepto de desprotección infantil y sus diferentes tipologías, y distinguían niveles de gravedad en las situaciones de desprotección, aportando algunos criterios generales para valorarlos. A pesar de que estos manuales fueron elaborados de forma independiente en cada territorio histórico, coincidían en los aspectos señalados y constituyeron un punto de partida importante para la elaboración del instrumento BALORA, pues aportaban un marco, un lenguaje y unos criterios generales de actuación compartidos.

\subsubsection{Búsqueda bibliográfica y documental (febrero- junio 2007)}

La primera actuación que se llevó a cabo para construir el instrumento BALORA fue una revisión bibliográfica y documental en bases de datos y organizaciones internacionales especializadas. La búsqueda se centró en criterios e instrumentos para la delimitación, definición y evaluación de la gravedad de las situaciones de desprotección infantil. Se prestó especial atención a la identificación de instrumentos con soporte teórico y empírico con objetivos similares a los pretendidos con el instrumento BALORA.

Se encontraron varios instrumentos que respondieron a ese propósito, ninguno en España. Entre ellos pueden citarse las Child Well-Being Scales -CWBS- (Magura y Moses, 1986), Ontario Child Neglect Index -CNI- (Trocmé, 1996), Modified Maltreatment Classification System -MMCS(English y the LONGSCAN Investigators, 1997), Graded Care Profile Scale -GCP- (Srivastava y Polnay, 1997), Parent-Child Conflict Tactics Scale CTSPC - (Straus y Hamby, 1997), Family Assessment Form -FAF- (Children's Bureau of Southern California, 1997), Severity Index (Slep y Heyman, 2004), y Eligibility Spectrum (Ontario Association of Children's Aid Societies, 2006). Estos instrumentos presentaban diferencias en las tipologías de desprotección recogidas, las conductas parentales y situaciones incluidas en cada una de ellas, el nivel de concreción con el que eran descritas, y el número, denominación y significado que otorgaban a los distintos niveles de gravedad que establecían a nivel general y en cada tipología. Sin embargo, todos ellos coincidían en varios aspectos importantes: a) incluían dos componentes principales para determinar la gravedad de la desprotección, por una parte, la conducta parental y, por otra parte, el daño o riesgo de daño que dicha conducta suponía para el desarrollo físico, cognitivo, social o emocional del niño, niña o adolescente; (b) asignaban un peso diferente a uno u otro componente en función de la tipología de desprotección; (c) tomaban en consideración la frecuencia e intensidad (no la intencionalidad) de la conducta parental para determinar su gravedad; (d) diferenciaban niveles de daño o riesgo de daño en el niño, niña o adolescente; y, (e) proponían descriptores específicos para cada nivel de gravedad en cada tipología de desprotección.

\subsubsection{Determinación y acuerdo sobre los fundamentos conceptuales del instrumento BALORA (junio-octubre 2007)}

La búsqueda bibliográfica y documental permitió elaborar una primera propuesta de lo que pretendían ser los fundamentos conceptuales del nuevo instrumento. En junio 2007, esta propuesta fue presentada a la comisión técnica, que la estudió y debatió en los meses siguientes, acordando dos cuestiones básicas:

1. En primer lugar, cuáles iban a ser los objetivos del instrumento y las situaciones en que debía aplicarse, algo que aunque a primera vista pudiera parecer obvio dado que estaba definido en la Ley $3 / 2005$, no lo era. La cuestión clave acordada a este respecto fue focalizar el trabajo en diseñar un instrumento para valorar la gravedad de situaciones de riesgo y desamparo vinculadas al ejercicio de los deberes de protección.

2. En segundo lugar, cuál iba a ser la definición del concepto de desprotección infantil y sus tipologías, su encaje con los conceptos legales de riesgo y desamparo, los componentes a tomar en consideración para valorar la gravedad de estas situaciones y el peso a asignar a cada componente, así como los niveles de gravedad a diferenciar, su significado y sus implicaciones para la intervención.

En esta primera fase se clarificó y acordó también terminología y un lenguaje común, cuestiones a menudo no suficientemente atendidas pero centrales en el instrumento y en el trabajo de los Servicios de Atención y Protección a la Infancia y Adolescencia.

\subsubsection{Elaboración del primer y segundo borradores (noviembre 2007-abril 2008)}

Tras acordar los fundamentos conceptuales del instrumento, se elaboró una propuesta de tipologías de desprotección, niveles de gravedad para cada una de ellas, y descriptores. La propuesta se basó principalmente en los criterios recogidos en las Child Well-Being Scales (Magura y Moses, 1986), el Eligibility Spectrum (Ontario Association of Children's Aid Societies, 2006) y el Modified Maltreatment Classification System (English y the LONGSCAN Investigators, 1997).

La propuesta fue presentada y analizada en detalle por la comisión técnica, hasta llegar a un consenso sobre el contenido de lo que fue el primer borrador 
del instrumento. Los miembros de la comisión técnica hicieron una aplicación piloto de este primer borrador con un total de 36 casos seleccionados de expedientes activos o recientes de sus servicios. Esta aplicación sirvió para hacer un primer estudio de fiabilidad interjueces. Con las cuestiones surgidas y resultados de esta primera aplicación, la comisión técnica volvió a revisar el instrumento, incluyéndose nuevas modificaciones que dieron lugar al segundo borrador. Esta fase finalizó en abril de 2008.

\subsubsection{Tercer y cuarto borrador: pilotaje y análisis de fiabilidad interjueces (mayo 2008-febrero 2009)}

En mayo de 2008, el segundo borrador del instrumento fue presentado a un "grupo piloto" formado por 24 profesionales voluntarios y voluntarias pertenecientes a las tres Diputaciones Forales y a los Ayuntamientos de Bilbao, Galdakao, Busturialdea, Donostia-San Sebastián, Irún, Arrasate y Vitoria-Gasteiz que en su trabajo cotidiano investigaban y valoraban sospechas o casos confirmados de desprotección infantil. Aplicaron el instrumento en sus servicios durante tres meses (de junio a septiembre de 2008). Sus valoraciones, aportaciones y dudas fueron recogidas y estudiadas por la comisión técnica. Se incluyeron nuevas modificaciones y se conformó el tercer borrador.

Este borrador fue sometido a una nueva prueba de fiabilidad interjueces con el grupo piloto. En base a los resultados obtenidos se incorporaron las últimas modificaciones al instrumento, llegando en febrero 2009 a su cuarto borrador o versión preliminar.

\subsubsection{Difusión generalizada de la versión preliminar del instrumento, implantación piloto y estudios empíricos (junio 2009-octubre 2010)}

Entre junio y septiembre de 2009 , se procedió a la difusión de la versión preliminar del instrumento entre el conjunto de servicios sociales municipales y territoriales de atención y protección a la infancia y adolescencia de la Comunidad Autónoma del Pasís Vasco, equipos concertados o contratados, y responsables y profesionales de otros servicios relacionados (por ejemplo, equipos psicosociales de los juzgados). La difusión se hizo en forma de sesiones de presentación, a las que asistieron un total de 724 profesionales: 202 en Álava, 373 en Bizkaia y 149 en Gipuzkoa. En total se celebraron cinco sesiones, cada una de ellas de cinco horas de duración. Además de estas sesiones, en el último trimestre del año 2009 se desarrollaron planes formativos complementarios en los tres territorios históricos.

Entre octubre de 2009 y octubre de 2010 se llevó a cabo la implantación piloto de la versión preliminar del instrumento BALORA con aquellos servicios y profesionales que voluntariamente quisieron hacerlo. Se puso en marcha un servicio de apoyo técnico telefónico y online para centralizar, recoger y atender dudas, comentarios y sugerencias con relación al instrumento. En este período, la comisión técnica se mantuvo en comunicación regular.

En mayo-junio de 2010 se remitió al conjunto de servicios sociales municipales y territoriales de atención y protección a la infancia y adolescencia un cuestionario dirigido a conocer el grado de utilización del instrumento y la valoración sobre su utilidad; la respuesta obtenida fue limitada (únicamente se recogieron setenta y cinco cuestionarios). La mayoría de los y las profesionales que respondieron expresaron un acuerdo mayoritario (cercano al $90 \%$ ) sobre la utilidad y necesidad del instrumento y unas expectativas positivas sobre su efecto en el logro de un mayor acuerdo entre servicios sociales municipales y territoriales en relación con la gravedad de los casos; sin embargo, informaron que solo una cuarta parte de sus servicios estaban utilizando el instrumento de forma sistemática.

En esta fase se llevaron a cabo varios estudios con el objetivo de evaluar el efecto del instrumento. Estos estudios, realizados con viñetas, constataron porcentajes elevados de desacuerdos y errores en la valoración de la gravedad de la desprotección realizada por los y las profesionales de los servicios de atención y protección a la infancia y adolescencia cuando utilizaban sus propios criterios. En el primero de estos estudios se recogieron datos de 515 de los y las 724 profesionales que participaron en las sesiones de presentación. Al inicio de la sesión y antes de disponer del instrumento BALORA, se presentaron a estos y estas profesionales 15 viñetas que describían situaciones de maltrato físico, negligencia en supervisión, maltrato emocional e incapacidad parental de control de la conducta infantil o adolescente de distinto nivel de gravedad. El nivel de gravedad de cada viñeta había sido acordado previamente por la comisión técnica. Se pidió a los y las 515 profesionales que leyeran las viñetas y calificaran el nivel de gravedad que les asignarían con los criterios que aplicaban hasta entonces en su trabajo cotidiano. Cada profesional calificó cuatro viñetas. Los resultados obtenidos fueron preocupantes. En ninguna de las viñetas se alcanzaron porcentajes de acuerdo y calificación correcta ni siquiera cercanos al $80 \%$, cifra considerada mínima. En términos globales, el porcentaje de profesionales que calificó correctamente el nivel de gravedad de las viñetas fue del $53 \%$ para las viñetas de maltrato físico, $48 \%$ para las viñetas de incapacidad parental de control de la conducta infantil o adolescente, $36 \%$ para las viñetas de maltrato emocional y $33 \%$ para las viñetas de negligencia en supervisión. Un 46,3\% de los y las profesionales no calificaron correctamente ninguna o solo una de las cuatro viñetas que se le proporcionaron. Los errores y desacuerdos en la calificación del nivel de gravedad se produjeron igualmente en todas las disciplinas profesionales, en profesionales de servicios sociales municipales y territoriales, y con más o menos años 
de experiencia. Los porcentajes de desacuerdo y errores descendieron significativamente en algunas de las viñetas al utilizar el instrumento BALORA aun con un conocimiento muy superficial de su contenido tras cinco horas de formación (Arruabarrena y De Paúl, 2011, 2012). Estudios posteriores encontraron relación entre un mayor conocimiento y dominio del instrumento y porcentajes más elevados de acuerdo y calificación correcta del nivel de gravedad (Arruabarrena, De Paúl, Indias y Ullate, 2013). Estos porcentajes llegaron al $72 \%-87 \%$ en un estudio realizado con 26 viñetas de negligencia y maltrato psíquico en el que participaron 39 profesionales especializados en protección infantil y con experiencia de un año de utilización del instrumento (Arruabarrena, datos no publicados).

\subsubsection{Redacción de la primera versión del instrumento Balora y aprobación como decreto (noviembre 2010-noviembre 2011)}

El conjunto de aportaciones recogidas en el período de implantación piloto del instrumento fueron estudiadas por la comisión técnica y utilizadas para la redacción en noviembre de 2010 de su última versión.

Un año después, en noviembre de 2011, y tras la tramitación del procedimiento oportuno, los trabajos descritos se materializaron en el Decreto 230/2011, de 8 de noviembre - publicado en el Boletín Oficial del País Vasco (BOPV) número 233, de fecha 12 de diciembre de 2011-. El decreto aprobó el instrumento BALORA y estableció su uso obligatorio en los servicios sociales municipales y territoriales de atención y protección a la infancia y adolescencia de la Comunidad Autónoma del País Vasco.

\subsection{Segunda fase: actualización del instrumento (2014-2017)}

\subsubsection{Revisión del proceso de implantación del instrumento y actualización de su contenido (abril 2014-febrero 2015)}

Transcurridos algo más de dos años desde la aprobación del instrumento BALORA, el Gobierno Vasco promovió un nuevo proceso dirigido a revisar en profundidad su grado de implantación, impacto y contenido. Las actividades llevadas a cabo en este proceso de revisión, completado entre abril de 2014 y febrero de 2015, incluyeron la elaboración y remisión de un cuestionario a la totalidad de responsables técnicos y equipos de los servicios sociales municipales y territoriales de atención y protección a la infancia y adolescencia, la realización de una nueva búsqueda bibliográfica y documental, y la organización y celebración de tres grupos de discusión (uno en cada territorio histórico) con profesionales representantes de las tres diputaciones forales, y los ayuntamientos de Bilbao, Leioa, Portugalete, Getxo, Galdakao, Berriz, Donostia-
San Sebastián, Irún, Azpeitia, Arrasate, Zarautz y Vitoria-Gasteiz.

Un total de 394 profesionales que utilizaban o potencialmente podían utilizar el instrumento BALORA en su ejercicio profesional cotidiano y 67 responsables técnicos contestaron al cuestionario. La mayoría de estos y estas profesionales llevaba tres o más años utilizándolo y había recibido formación sobre su uso (aunque un porcentaje cercano al $20 \%$-la mayor parte profesionales de nueva incorporación- no la había recibido y consideraba que la necesitaba). El $70 \%$ utilizaba el instrumento de forma habitual (más de una vez al mes), el $80 \%$ lo hacía cuando tenía que valorar un caso de desprotección o posible desprotección (habiendo un $20 \%$ de profesionales que no lo hacía) y el $95 \%$ lo utilizaba también en otros momentos del proceso de intervención. Más del $95 \%$ de los y las profesionales consideraba que el instrumento era útil y necesario. El $90 \%$ opinaba que contribuía a un mayor acuerdo entre servicios sociales municipales y territoriales respecto a la gravedad del caso y la institución a la que correspondía atenderlos.

Las respuestas de los y las 67 responsables técnicos que contestaron al cuestionario coincidieron en una valoración positiva y porcentajes similares a los y las profesionales con relación a la utilidad, necesidad y contribución del instrumento BALORA a un mayor acuerdo entre servicios sociales municipales y territoriales respecto a la gravedad de los casos y la institución a la que correspondía atenderlos. Aproximadamente la mitad de los y las responsables técnicos informó que había profesionales en sus servicios que necesitaban formación sobre el instrumento.

Los cuestionarios preguntaron también sobre los aspectos positivos o beneficios principales del instrumento (respondieron 293 profesionales y 41 responsables técnicos), así como sus limitaciones y las dificultades principales para su utilización (respondieron 272 profesionales y 41 responsables técnicos). Por último, se preguntó sobre propuestas de modificación o mejora del instrumento (se recogieron propuestas de 152 profesionales y 25 responsables técnicos). La información cuantitativa y cualitativa obtenida a través de los cuestionarios se analizó con mayor detalle en los grupos de discusión organizados en cada territorio histórico.

Entre noviembre de 2014 y marzo de 2015, la comisión técnica, que mantuvo la misma composición que en fases previas, procedió al análisis pormenorizado de las propuestas de modificación del instrumento recogidas de los cuestionarios y los grupos de discusión, las recibidas a través de otros canales, y las realizadas por la propia comisión. Algunas de las propuestas de modificación hacían referencia a la primera parte del instrumento (por ejemplo, explicitación de la inserción del instrumento en el proceso de intervención de los servicios de atención y protección a la infancia y adolescencia y 
en los manuales técnicos de actuación disponibles en cada territorio histórico, mayor precisión en la definición de "daño significativo", "daño psíquico" y “daño grave”, inclusión de criterios técnicos más precisos sobre la actuación a seguir por los servicios sociales municipales en casos de sospechas no confirmadas de desprotección de gravedad elevada o muy elevada); otras implicaban cambios en las escalas del instrumento (por ejemplo, ampliación de la escala de instrumentalización en conflictos entre las figuras parentales a situaciones análogas de instrumentalización en conflictos entre las figuras parentales y otros familiares significativos para el niño, niña o adolescente pertenecientes al núcleo convivencial, inclusión de nuevos descriptores en algunas escalas), mientras que otras modificaciones afectaban a la hoja-resumen del instrumento o a la ampliación de los anexos. Se incluyeron también precisiones técnicas varias de carácter secundario, correcciones ortográficas, terminológicas y de redacción, y se procedió a la eliminación de contenidos reiterativos.

\subsubsection{Adecuación del instrumento a cambios legislativos en materia de protección a la infancia y adolescencia y servicios sociales (abril 2016-abril 2017)}

Poco tiempo después de concluir la fase anterior, en julio de 2015, se aprobaron la Ley Orgánica 8/2015, de 22 de julio, y la Ley $26 / 2015$, de 28 de julio, ambas de modificación del sistema de protección a la infancia y a la adolescencia. Las citadas leyes conllevaron una revisión y modificación de las instituciones jurídicas de protección a la infancia y adolescencia contempladas en el ordenamiento jurídico español y establecieron un marco normativo común en relación a determinadas cuestiones que carecían de regulación básica o desarrollo normativo. En particular, la Ley 26/2015 introdujo modificaciones en terminología técnico-jurídica, así como en la regulación de las situaciones de riesgo y de desamparo, $y$, en concreto, en las actuaciones a realizar ante dichas situaciones por parte de las entidades públicas competentes en materia de protección de menores. Ese mismo año tuvo lugar la aprobación del Decreto 185/2015, de 6 de octubre, de cartera de prestaciones y servicios del Sistema Vasco de Servicios Sociales, y de la Ley 39/2015, de 1 de octubre, del Procedimiento Administrativo Común de las Administraciones Públicas.

Entre abril y octubre de 2016, la comisión técnica que desde 2007 había trabajado en la elaboración y posterior actualización del instrumento BALORA comenzó una nueva fase de trabajo para la adecuación del instrumento a los cambios legislativos y normativos anteriormente referidos. Fruto de este trabajo se incluyeron, además de ajustes a nivel de lenguaje técnico-jurídico, cambios de contenido en el instrumento entre los que cabe destacar los siguientes:
1. La aportación de criterios técnicos para la decisión sobre la declaración administrativa de la situación de riesgo, estableciéndose que se procediera de esta forma en las situaciones de riesgo moderado cercano a grave y de riesgo grave cercano a desamparo donde se evidenciara falta de colaboración por parte de los padres y madres o responsables legales del niño, niña o adolescente. El instrumento BALORA incluyó además criterios técnicos para la valoración del referido grado de colaboración.

2. Una mayor atención sobre la obligación de promover la participación y consideración de la opinión de los padres y madres o responsables legales y de los niños, niñas y adolescentes si tuvieren suficiente madurez y siempre a partir de los doce años, en la elaboración de los planes de intervención y los procesos de toma de decisión.

3. La alusión explícita a las situaciones de riesgo prenatal y a la importancia de las intervenciones de carácter preventivo y precoz en éstas y otras situaciones de vulnerabilidad detectadas en el período prenatal, la aportación de criterios técnicos para su identificación, y la inclusión de indicaciones sobre la actuación a seguir en estos casos.

En ocubre de 2016, la versión actualizada del instrumento fue remitida a la asesoría jurídica del Departamento de Empleo y Políticas Sociales del Gobierno Vasco para la emisión del correspondiente informe preceptivo. Sus consideraciones fueron estudiadas, valoradas y aceptadas por la comisión técnica, incluyéndose las oportunas modificaciones en el instrumento. El 21 de noviembre de 2016 se inició formalmente el procedimiento para la elaboración del proyecto de decreto dirigido a la actualización del instrumento BALORA. En este contexto fue presentado, analizado, sometido a informe y aprobado por la Comisión Permanente Sectorial para la Atención a la Infancia y Adolescencia. Asimismo, fue sometido al correspondiente trámite de audiencia y participación y consulta a las administraciones públicas: con EUDEL -en su calidad de asociación de municipios más representativa a nivel de la Comunidad Autónoma-, con todos los ayuntamientos y con las diputaciones forales. Las aportaciones recogidas en el marco de estos trámites fueron estudiadas por la comisión técnica, incluyéndose aquellas que fueron consideradas oportunas y desestimándose de forma argumentada en caso contrario.

El proceso de actualización del instrumento BALORA finalizó en abril de 2017, con la aprobación del Decreto 152/2017, de 9 de mayo, publicado en el BOPV, número 125, de fecha 3 de julio de 2017. En el BOPV, número 136, de fecha 18 de julio 2017, se publicó una corrección de errores del citado decreto con motivo de un error de paginación que dió lugar a su reproducción íntegra. 


\section{Fundamentos conceptuales}

Conocer los fundamentos conceptuales del instrumento BALORA es imprescindible para poder utilizarlo correctamente. Se describen en su primera parte (apartados "Parte I: Situaciones de riesgo y desamparo: Definición y niveles de gravedad” y "Parte II: Criterios generales y procedimiento para la valoración de la gravedad de las situaciones de riesgo y desamparo"). Su lectura detallada y asimilación deberían ser requisitos ineludibles para acceder al instrumento propiamente dicho (escalas). Si hubiera de prescindirse de alguna parte del instrumento, nunca debería ser la primera.

A continuación, se describen los fundamentos principales que sustentan la construcción del instrumento BALORA:

- El instrumento no es de aplicación en todas las situaciones de riesgo. Su objetivo es ayudar en la valoración de la gravedad de las situaciones vinculadas al ejercicio de los deberes de protección por parte de los padres y madres o responsables legales de un niño, niña o adolescente. Por tanto, deja fuera de su propósito las situaciones de riesgo (denominadas situaciones de "dificultad social") en las que, habiendo un ejercicio adecuado de los deberes de protección, existen otras circunstancias, carencias o conflictos familiares, sociales o educativos fuera del control de los padres y madres o responsables legales que perjudican o pueden perjudicar el desarrollo del niño, niña o adolescente (por ejemplo, entornos sociales con imágenes identificatorias no deseables, falta de servicios, pobreza, alto índice de delincuencia, marginación o violencia en el entorno comunitario, violencia en el ámbito escolar). Si bien los servicios sociales también han de intervenir en estos casos, los criterios en base a los cuales se ha de valorar su gravedad y determinar la intervención a seguir son distintos de los criterios a utilizar en casos vinculados al ejercicio de los deberes de protección, y, por tanto, requieren instrumentos técnicos diferentes.

- En consonancia con lo anterior, el instrumento BALORA se diseñó con el objetivo exclusivo de valorar la presencia y gravedad de los déficits en el ejercicio de los deberes de protección por parte de los padres y madres o responsables legales. El instrumento no pretende servir para valorar la gravedad de las dificultades de los niños, niñas o adolescentes, la gravedad de los problemas familiares causantes o mantenedores de la situación de riesgo o desamparo, o la gravedad de otros problemas externos a la familia. Se trata de valoraciones diferentes, que implican la toma en consideración de información y criterios diferentes, y por tanto requieren instrumentos técnicos también diferentes.

- El instrumento se centra en "qué” le ha pasado al niño, niña o adolescente y la gravedad de lo ocurrido, no en "por qué" ha pasado. Esta diferenciación es importante. Desde la perspectiva del niño, niña o adolescente (que es donde se sitúa el instrumento BALORA), es igualmente grave la situación de un niño de cinco años de familia monoparental que es dejado con frecuencia solo en el domicilio familiar durante parte de la noche porque su madre, que le quiere, cuida y trata adecuadamente, ha de salir a trabajar, que si el mismo niño fuera dejado solo por una madre inmadura y centrada en sus propias necesidades, que sale por la noche a relacionarse con jóvenes de su edad y su nueva pareja. La madre del primer caso, además, se muestra consciente del riesgo en el que coloca a su hijo y de que no debería hacerlo, pero necesita los ingresos económicos y carece de apoyos, mientras que la madre del segundo caso resta importancia al peligro en el que puede encontrarse su hijo. El riesgo que corren estos dos niños (accidente doméstico, urgencia médica, etc.) es el mismo en ambos casos, es decir, no hay diferencias en la gravedad y lo peligroso del hecho de dejar a un niño de esta edad solo durante periodos prolongados de tiempo. En ambas situaciones deberían intervenir los servicios de atención y protección a la infancia y adolescencia, $y$, de acuerdo con los criterios del instrumento BALORA correspondería hacerlo a los servicios sociales territoriales. Ahora bien, la intervención con ambos casos será totalmente diferente, básicamente porque las causas y factores asociados son distintos y el pronóstico del caso también parece serlo. El primero de ellos puede ser de fácil y rápida resolución, mientras que el segundo previsiblemente presentará mayores dificultades pudiendo llegar a ser necesaria la declaración de desamparo del niño. La gravedad de la desprotección se evalúa fundamentalmente en función del impacto que el comportamiento de los padres y madres o responsables legales tiene o puede tener en el niño, niña o adolescente, no en función del tipo, número o gravedad de los problemas que afectan a la familia.

El ejemplo anterior ilustra la necesidad de diferenciar entre "qué" ha pasado (y cómo ha sido de grave) y "por qué”. Y también muestra la necesidad consecuente de diferenciar entre la gravedad de la situación que ha afectado a un niño, niña o adolescente (gravedad de la desprotección) y el número o gravedad de los problemas familiares y la dificultad para la intervención, que no son equivalentes ni correlacionan de forma automática. Si bien es cierto que existe una asociación entre estas variables (en general, los casos de desprotección más grave tienden a presentar mayor número y severidad de problemas en la familia y por tanto mayor dificultad para la intervención), no siempre es así. Clara constatación de ello son los numerosos casos de desprotección de gravedad moderada (particularmente negligencia crónica) atendidos en los servicios sociales municipales realmente difíciles y resistentes a la intervención. 
- El instrumento BALORA describe las distintas tipologías o formas en que se pueden manifestar las situaciones de riesgo o desamparo vinculadas al ejercicio de los deberes de protección. Ese listado pretende ser exhaustivo, aunque pudiera haber situaciones excepcionales no recogidas 0 situaciones que puedan incorporarse en un futuro por cambios en los usos y normas sociales (como ha sucedido, por ejemplo, con la exposición de los niños, niñas o adolescentes a conductas de riesgo vinculadas a la seguridad vial o a las nuevas tecnologías).

Para valorar la gravedad de las situaciones de riesgo y desamparo, el instrumento BALORA toma en cuenta exclusivamente dos componentes:

a. El comportamiento parental, concretamente su tipo (que ha de corresponder a alguna de las tipologías descritas en el instrumento), frecuencia e intensidad.

b. El daño que dicho comportamiento ha provocado (daño real) o puede provocar (daño potencial) en el niño, niña o adolescente a nivel físico o psíquico (áreas emocional, cognitiva, social y sexual).

Es la combinación de estos dos componentes lo que determina la gravedad de la situación del niño, niña o adolescente. El instrumento requiere que la presencia de ambos componentes quede probada con evidencias objetivables, 0 cuando menos suficientemente argumentada y justificada. Si eso no se produce con alguno de estos componentes, no se podrá concluir sobre el nivel de gravedad. Se exceptúan algunos comportamientos parentales de contenido inherentemente traumático (por ejemplo, abuso sexual) o que afectan a niños, niñas o adolescentes particularmente vulnerables (por ejemplo, de corta edad o con discapacidades), en los que el instrumento exime de la necesidad de demostrar, justificar o argumentar el segundo de los componentes, esto es, el daño en el niño, niña 0 adolescente porque asume que se está produciendo aunque no se manifieste y que es significativo. En el resto de casos el instrumento requiere realizar una evaluación de cada componente en cada caso concreto. Esto es muy importante, particularmente en lo relativo al daño real o potencial en el niño, niña o adolescente, pues el mismo comportamiento parental puede ser calificado con distinto nivel de gravedad o incluso no ser calificado como desprotección según su impacto. $Y$ tener en cuenta el impacto en el niño, niña o adolescente afectado supone estar considerando implícitamente variables como su edad, historia previa, sus características personales, la presencia de otras experiencias adversas o factores de protección en su entorno, o su percepción del comportamiento parental (intencionalidad, componente afectivo).

- El instrumento BALORA contempla el ejercicio de los deberes de protección como un continuo en cuyos extremos se sitúan, por una parte, el ejercicio idóneo y, por otra, el ejercicio extremadamente inadecuado del rol parental. Este último conduciría a la declaración de desamparo. En este continuo se diferencian las siguientes situaciones, todas ellas objeto de atención de los servicios sociales de atención y protección a la infancia y adolescencia: vulnerabilidad a la desprotección, riesgo leve, riesgo moderado, riesgo grave y desamparo (ver Figura 1). Las situaciones de vulnerabilidad a la desprotección, riesgo leve y riesgo moderado corresponden ser atendidas por los servicios sociales municipales, mientras que las situaciones de riesgo grave y desamparo son competencia de los servicios sociales territoriales.

\section{Figura 1. Situaciones a diferenciar en el continuo del ejercicio de los deberes de protección}

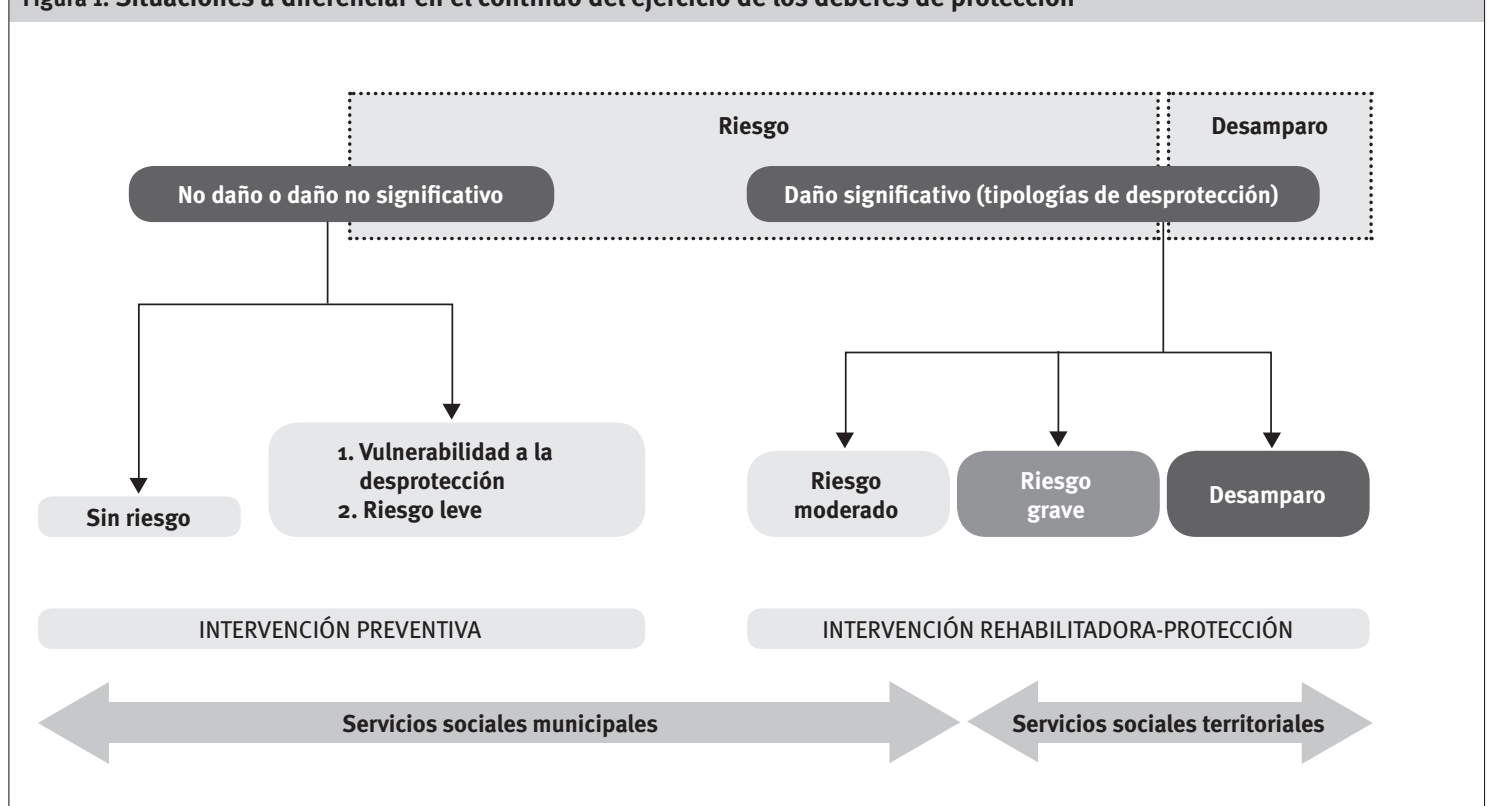


Es importante detenerse en los criterios que utiliza el instrumento BALORA para establecer los puntos de corte entre estas categorías. No es algo sencillo, pues se trata, como se ha señalado anteriormente, de un continuo.

a. El primer punto de corte en sentido ascendente se sitúa entre las situaciones de vulnerabilidad a la desprotección y el resto (riesgo y desamparo). Lo que establece la diferencia es que en las situaciones de riesgo y desamparo hay un comportamiento parental inadecuado o incumplimiento de los deberes de protección, y como consecuencia de ello el niño, niña o adolescente sufre o puede sufrir daño. En las situaciones de vulnerabilidad a la desprotección, la primera condición no está presente. La vulnerabilidad a la desprotección incluye situaciones en las que no ha habido una atención inadecuada a las necesidades del niño, niña o adolescente, pero hay antecedentes o una situación de crisis, déficits o problemas en la familia que se prevé que pueden afectar negativamente o limitar de forma significativa la capacidad de las figuras parentales para proporcionar un cuidado y atención adecuados al niño, niña o adolescente y provocar la aparición en un futuro próximo de situaciones de desprotección.

b. El segundo punto de corte se encuentra entre las situaciones de riesgo leve y el resto (riesgo moderado, riesgo grave y desamparo). La diferencia aquí radica en la gravedad del daño sufrido o que puede sufrir el niño, niña o adolescente. La calificación de riesgo moderado, riesgo grave o desamparo requiere que el daño sea significativo. Técnicamente este sería el límite a partir del cual calificar la existencia de desprotección. En este sentido, si bien las situaciones de riesgo moderado implican un daño menor que las situaciones de riesgo grave o desamparo, no son en ningún caso menos preocupantes ni implican una menor necesidad de intervención.

El punto de corte establecido por el criterio del "daño significativo" implica que en la categoría de riesgo leve se incluyen comportamientos adultos que pueden calificarse como inadecuados o no idóneos, pero que no conllevan daño significativo para el niño, niña o adolescente. Se trata de comportamientos frecuentes en muchos padres y madres (por ejemplo, insuficiencia de control hacia el uso de las nuevas tecnologías, inconsistencia o laxitud en normas y límites, consumo de alcohol o tabaco frente a los niños, niñas o adolescentes) que constituyen modelos o comportamientos no idóneos, que incluso pueden provocar daño al niño, niña 0 adolescente, pero que al no ser intensos 0 frecuentes no provocan ni se prevé que vayan a provocar un daño significativo. Por supuesto que estos comportamientos deberían ser eliminados, y los servicios sociales deben trabajar para ello a través de actividades y campañas preventivas en las que hagan conscientes a los padres y madres de la no conveniencia de estos comportamientos y les ofrezcan otros alternativos.

- El tercer y último punto de corte se encuentra entre las situaciones de riesgo moderado y el resto (riesgo grave y desamparo). La diferencia aquí radica nuevamente en la gravedad del daño sufrido o que puede sufrir el niño, niña o adolescente. La calificación de riesgo grave o desamparo requiere que el daño no sólo sea significativo, sino también grave.

El instrumento BALORA aporta criterios para determinar cuándo hay daño, cuándo es significativo y cuándo es grave. La actualización del instrumento incluyó, entre otros, una mayor concreción de los criterios para la evaluación y determinación de la gravedad del daño psíquico, un importante desafío para los y las profesionales de la salud mental particularmente en lo relativo a la argumentación de daño potencial en ausencia de síntomas de daño real y al establecimiento de nexos causales o vínculos entre el comportamiento parental y los síntomas de daño en el niño, niña o adolescente.

El instrumento recoge las distintas formas en que se puede manifestar la incapacidad, imposibilidad y el inadecuado ejercicio de los deberes de protección. Estas últimas son las tipologías de desprotección infantil, que incluyen tanto la perpetración directa de comportamientos inadecuados (de carácter físico, psicológico o sexual; por acción u omisión) por parte de los padres, madres o responsables legales, como su incapacidad para proteger al niño, niña o adolescente de situaciones de trato gravemente inadecuado perpetrado por otras personas -adultas o menores de edad-. El instrumento distingue así 27 tipologías de desprotección (ver Tabla 1).

Para cada tipología de desprotección, el instrumento describe los niveles de riesgo leve, gravedad moderada, gravedad elevada y gravedad muy elevada (salvo excepciones en las que no se contempla la existencia de algún nivel de gravedad) y proporciona una descripción en forma de párrafo de las situaciones incluidas en cada uno de ellos. Los niveles de gravedad se determinan, como se ha señalado anteriormente, tras el análisis y valoración combinada de dos componentes: el comportamiento parental y el daño real o potencial en el niño, niña o adolescente. Obviamente, a la hora de aplicar el instrumento en casos concretos no se espera que todos los descriptores que conforman un nivel de gravedad se presenten en su literalidad. Para calificar un determinado nivel de gravedad es suficiente con que se presente la parte sustancial de los descriptores recogidos y que las características del caso evaluado se ajusten a lo que dichos descriptores pretenden reflejar. Esto es lo que el o la profesional que utiliza el instrumento deberá argumentar y justificar. 
Maltrato físico (1)

Negligencia (14)

A. Negligencia hacia necesidades físicas - 6 escalas: alimentación; cuidado de la salud física; vestido; higiene personal; condiciones higiénicas de la vivienda; estabilidad y condiciones de habitabilidad de la vivienda.

B. Negligencia hacia necesidades de seguridad - 3 escalas: seguridad física de la vivienda y prevención de riesgos; supervisión; protección ante situaciones de desprotección grave perpetradas por otras personas.

C. Negligencia hacia necesidades formativas -1 escala.

D. Negligencia hacia necesidades psíquicas - 4 escalas: necesidades de interacción y afecto; estimulación; atención específica a problemas emocionales graves; normas, límites y transmisión de valores morales positivos.

Abuso sexual (1)

Maltrato psíquico (4)

A. Maltrato emocional.

B. Instrumentalización en conflictos entre las figuras parentales y conflictos entre las figuras parentales y otros familiares significativos para el niño, niña o adolescente pertenecientes al núcleo convivencial.

C. Exposición a situaciones de violencia de pareja o entre miembros de la unidad convivencial.

D. Amenazas de agresión física.

Abandono (1)

Incapacidad parental de control de la conducta del niño, niña o adolescente (1) Otras (5)

A. Maltrato prenatal.

B. Trato inapropiado (incluye privación deliberada de comida y agua, confinamiento o restricción física, expulsión o negación de la entrada al domicilio).

C. Corrupción.

D. Mendicidad.

E. Explotación laboral.

- El instrumento BALORA ayuda a los y las profesionales a identificar las tipologías de desprotección presentes en cada caso, valorar la gravedad individual de cada una de ellas (riesgo leve, gravedad moderada, gravedad elevada, gravedad muy elevada), y finalmente concluir en una valoración global del nivel de gravedad del caso: sin riesgo, riesgo leve, riesgo moderado, riesgo grave, desamparo. Estas valoraciones se llevan a cabo de acuerdo a los siguientes criterios:

a. La presencia de un indicador de gravedad muy elevada o gravedad elevada en cualquiera de las tipologías supone, independientemente de la gravedad del resto de indicadores, la valoración global del caso como riesgo grave o desamparo, lo que implica que la intervención es competencia de los servicios sociales territoriales. Esto será así independientemente de la actitud y disposición de la familia hacia la intervención. Serán los servicios sociales territoriales quienes, con el conjunto de la información recabada sobre el niño, niña o adolescente y su familia, determinen si se trata de una situación de riesgo grave o de desamparo.

b. La presencia de al menos un indicador de gravedad moderada sin que existan indicadores de gravedad elevada o muy elevada, supone la valoración de la desprotección global del caso como riesgo moderado. Salvo excepciones debidamente argumentadas, la presencia simultánea de varios indicadores de gravedad moderada no incrementa el nivel de gravedad global del caso. c. La presencia de indicadores de riesgo leve sin que existan indicadores de gravedad moderada, elevada o muy elevada, supone la valoración global del caso como riesgo leve. Tampoco en este caso, salvo excepciones debidamente argumentadas, la presencia simultánea de varios indicadores de gravedad leve incrementa el nivel de gravedad global del caso a riesgo moderado. La intervención en estos casos corresponde a los servicios sociales municipales.

- El instrumento BALORA otorga un papel importante a la colaboración de los padres y madres o responsables legales con la intervención de los servicios sociales en la determinación del nivel global de gravedad de la desprotección y en la toma de decisión sobre la intervención a seguir. Esta importancia se acrecenta en la actualización que se produce tras la aprobación de la Ley 26/2015, de 28 de julio, de modificación del sistema de protección a la infancia y a la adolescencia, llegando a incluirse en el instrumento criterios técnicos para evaluar y argumentar el grado de colaboración. Entre otras cosas, el instrumento establece que el grado de colaboración de los padres, madres o responsables legales con la propuesta de intervención de los servicios sociales, serán elementos que -además de la presencia o no de indicadores de desprotección y su gravedadsiempre deberán ser valorados antes de determinar el nivel global de gravedad de un caso.

- El trabajo en los servicios de atención y protección a la infancia y adolescencia implica tomas de decisión continuas, diversas e ineludibles. 
Entre ellas se incluyen, por ejemplo, si se inicia la intervención de los servicios de atención y protección a la infancia y adolescencia, si el caso ha de ser atendido por los servicios sociales municipales o territoriales, el nivel de urgencia con el que hay que actuar, si el niño, niña o adolescente ha de ser separado de su familia, si la separación ha de ser temporal o definitiva, si puede retornar, qué recursos han de aplicarse en el caso, si deben mantenerse o no, etc. Se trata de decisiones de gran impacto y que pueden ser determinantes en la vida de los niños, niñas y adolescentes y sus familias. Cada una de esas decisiones requiere la toma en consideración de información diferente. Incluso la misma variable puede tener distinto peso según la toma de decisión. Lo relevante en relación al instrumento BALORA es que:

a. La presencia y gravedad de la desprotección, que es lo que evalúa este instrumento, es una variable central o relevante en la mayoría de estas decisiones. Esto significa que el instrumento no será de aplicación únicamente en las primeras fases de la intervención de los servicios sociales (primera valoración tras la notificación, fases de investigación y evaluación), sino que habrá de serlo a lo largo de todo el proceso de intervención: desde la recepción de una notificación hasta la finalización de la intervención y el cierre del expediente.

b. No obstante, la gravedad de la desprotección nunca es la única variable implicada en ninguna toma de decisión. Siempre hay otras variables a considerar, que deberán ser valoradas conjuntamente para conducir a la toma de decisión. De ahí que la aplicación del instrumento BALORA nunca pueda conducir de forma automática a una toma de decisión. Un claro ejemplo de ello es la declaración del desamparo y separación del niño, niña 0 adolescente de la familia. El instrumento BALORA llevará a concluir si la desprotección que afecta a un niño, niña o adolescente es de gravedad elevada o muy elevada, pero no llevará a concluir si tiene que ser separado o no de su familia. ¿Por qué? Porque en la toma de decisión sobre la separación entran en consideración otras variables además de la gravedad de lo que ha sucedido (por ejemplo, el grado de colaboración de los padres, madres o responsables legales con los servicios sociales). De hecho, la decisión de separar a un niño o niña de su familia se adopta no tanto por la gravedad de lo que ha pasado (que es en lo que se centra el instrumento BALORA), sino por la gravedad de lo que puede suceder en el futuro (lo que se denomina risk assessment). Y no siempre la gravedad de lo que ha sucedido predice la gravedad de lo que va a suceder. Puede darse un caso de maltrato físico de gravedad extrema en el que el niño o niña pueda mantenerse en su familia al valorarse que hay colaboración suficiente y factores de protección en el entorno que aseguran que no hay riesgo de repetición. Por el contrario, podría presentarse otro caso de maltrato físico de gravedad elevada (no extrema) en el que finalmente se decrete un desamparo al valorar que existe un riesgo importante de que el niño o niña vuelva a ser agredido y de que la gravedad del maltrato aumente. Así pues, aunque existe correspondencia entre gravedad moderada-riesgo moderado, entre gravedad elevada-riesgo grave y entre gravedad muy elevada-desamparo en una parte importante de los casos, la asociación no es automática.

\section{Contenido y estructura}

El instrumento BALORA se divide en tres partes: Objetivos, fundamentación y procedimiento para su utilización; escalas; y criterios técnicos y documentación complementaria.

\subsection{Primera parte: objetivos, fundamentación y procedimiento para la utilización del instrumento}

Como se ha señalado anteriormente, la utilización correcta del instrumento requiere necesariamente entender y dominar esta primera parte. Incluye los contenidos que recoge la Tabla 2.

\subsection{Segunda parte: escalas}

Esta parte del instrumento describe los aspectos a considerar para valorar la gravedad de cada una de las 27 tipologías de desprotección infantil, los descriptores de cada nivel de gravedad, y, en su caso, cuestiones particulares a tener en cuenta. Salvo excepciones en que no existe algún nivel de gravedad (por ejemplo, en la escala de abuso sexual no existe gravedad moderada o riesgo leve), cada tipología diferencia cinco niveles: no presente, riesgo leve, gravedad moderada, gravedad elevada y gravedad muy elevada.

El instrumento incluye también lo que denomina "Otras situaciones específicas que constituyen riesgo grave o desamparo". Se trata de situaciones en las que hay circunstancias de carácter extremo que implican un serio peligro para la vida o integridad básicas del niño, niña o adolescente, no existiendo elementos suficientes de control en el entorno familiar que puedan suplir los déficits presentes en la familia y garantizar una atención mínimamente adecuada a las necesidades básicas del niño, niña o adolescente. En la mayor parte de estos casos, en el momento de la valoración no se identifica ninguna tipología de desprotección, pero se valora que el riesgo de que suceda y de que tenga consecuencias muy graves para el niño, niña o adolescente es muy elevado. De acuerdo al instrumento, la presencia 
Tabla 2. Contenido de la primera parte del instrumento BALORA

\begin{tabular}{|c|c|}
\hline \multicolumn{2}{|l|}{ Contextualización del instrumento } \\
\hline \multicolumn{2}{|c|}{$\begin{array}{l}\text { Objetivos. Inserción en el procedimiento de intervención de los Servicios de atención y protección a la infancia y adolescencia; momentos de } \\
\text { aplicación. Pautas generales de utilización. }\end{array}$} \\
\hline \multicolumn{2}{|c|}{ Parte I. Situaciones de riesgo y desamparo: definición y niveles de gravedad } \\
\hline \multicolumn{2}{|c|}{$\begin{array}{l}\text { Concepto de desprotección: diferenciación de situaciones de no riesgo, vulnerabilidad a la desprotección, riesgo leve, riesgo moderado, } \\
\text { riesgo grave, desamparo. } \\
\text { Componentes de la definición de la desprotección: (1) comportamiento de los padres y madres o responsables legales, y (2) impacto actual o } \\
\text { potencial en el niño, niña o adolescente (daño significativo). }\end{array}$} \\
\hline \multicolumn{2}{|c|}{ Parte II. situaciones de riesgo y desamparo: valoración de la gravedad. Criterios generales y procedimiento } \\
\hline $\begin{array}{l}\text { Periodos de tiempo. Niveles de } \\
\text { gravedad, calificación y significado }\end{array}$ & $\begin{array}{l}\text { Periodo de tiempo que ha de abarcar la valoración. Procedimiento para calificar el nivel de gravedad } \\
\text { de cada tipología. Procedimiento para calificar el nivel global de gravedad del caso. Valoración de la } \\
\text { colaboración de la familia con la propuesta de intervención. Consideración de otras dificultades que } \\
\text { afectan a la familia y de situaciones de desprotección ocurridas en el pasado. }\end{array}$ \\
\hline $\begin{array}{l}\text { Concepto de "daño significativo"y } \\
\text { evaluación del daño psíquico }\end{array}$ & $\begin{array}{l}\text { Definición de “daño significativo”. Criterios para la evaluación de la presencia y gravedad de daño } \\
\text { psíquico en el niño, niña o adolescente. }\end{array}$ \\
\hline Circunstancias particulares & $\begin{array}{l}\text { Aplicación del instrumento en casos de parejas separadas o divorciadas, prácticas vinculadas a } \\
\text { valores o costumbres particulares; adolescentes no emancipados legalmente que viven de forma } \\
\text { independiente. }\end{array}$ \\
\hline $\begin{array}{l}\text { Procedimiento para la recogida de } \\
\text { información }\end{array}$ & $\begin{array}{l}\text { Áreas a recabar información. Valoración de aspectos afectivo-relacionales y de la afectación del } \\
\text { desarrollo psíquico (emocional, social, cognitivo y sexual) del niño, niña o adolescente: importancia y } \\
\text { procedimiento a seguir. Fuentes de información a consultar. Actuación a seguir cuando no es posible } \\
\text { recoger información suficientemente completa. }\end{array}$ \\
\hline $\begin{array}{l}\text { Servicios implicados y recursos } \\
\text { necesarios }\end{array}$ & Profesionales y servicios implicados en la valoración de una situación de desprotección y su gravedad. \\
\hline Elaboración del informe & Contenido general del informe de valoración. Utilización de la hoja-resumen del instrumento. \\
\hline $\begin{array}{l}\text { Derivación de casos entre servicios } \\
\text { en función de los resultados de la } \\
\text { valoración/Derivación de casos con } \\
\text { declaración de riesgo }\end{array}$ & $\begin{array}{l}\text { Momentos en que puede producirse la derivación. Servicios responsables de llevar a cabo la } \\
\text { valoración de la gravedad. Actuación en situaciones de urgencia. Condiciones y procedimiento } \\
\text { para la derivación de casos de servicios sociales municipales a territoriales; actuación en casos de } \\
\text { sospecha. condiciones y procedimiento para la derivación de casos de servicios sociales territoriales } \\
\text { a municipales; condiciones para la constatación de una disminución del nivel de gravedad de la } \\
\text { desprotección. Actuación a seguir cuando existen discrepancias. Actuación a seguir con niños, niñas y } \\
\text { adolescentes en la familia afectados por situaciones de diferente nivel de gravedad. } \\
\text { Particularidades del procedimiento de derivación de casos en los que se ha procedido a la declaración } \\
\text { de la situación de riesgo. }\end{array}$ \\
\hline Intervención posterior & $\begin{array}{l}\text { Líneas generales del diseño y ejecución del plan de intervención en situaciones de riesgo moderado, } \\
\text { riesgo grave o desamparo. }\end{array}$ \\
\hline
\end{tabular}

de estas circunstancias supone automáticamente la calificación de la gravedad del caso como elevada. La intervención corresponderá a los servicios sociales territoriales, quienes deberán determinar si se trata de una situación de riesgo grave o de desamparo.

\subsection{Tercera parte: criterios técnicos y documentación complementaria}

En su última parte, el instrumento BALORA aporta criterios técnicos relacionados con dos tipos de circunstancias, ambas destacadas en las modificaciones legislativas incluidas en la Ley 26/2015: la definición e intervención en situaciones de riesgo prenatal y vulnerabilidad a la desprotección detectada en el período prenatal, y la valoración responsables legales.

Por último, el instrumento incluye un glosario, una hoja-resumen que recoge de manera esquemática la información recabada tras la aplicación del instrumento y sus conclusiones (esta hoja-resumen deberá acompañar al informe descriptivo), una taxonomía de necesidades básicas en la infancia y adolescencia e indicadores para su evaluación (como apoyo para la valoración de la gravedad, particularmente útil en casos de negligencia), y una guía rápida diseñada para proporcionar una visión de conjunto del contenido del instrumento de forma ágil.

\section{Consideraciones finales}

El instrumento BALORA se diseñó con el objetivo de ayudar a los y las profesionales de los servicios de atención y protección a la infancia y adolescencia en la identificación y valoración de la gravedad de las situaciones de riesgo y desamparo. Se trataba de homogeneizar criterios, reducir errores y favorecer una mayor agilidad y calidad en los procesos de toma de decisión e intervención en estos casos. En el momento actual, su implantación en la Comunidad Autónoma del País Vasco es generalizada, hecho derivado sin duda de su aprobación como decreto y la consiguiente obligatoriedad en su utilización. Los estudios cuantitativos y cualitativos realizados 
hasta la fecha indican que el instrumento ha sido útil para los objetivos con los que fue diseñado y su solidez como instrumento técnico ha hecho que haya sido tomado como modelo o implantado en otras comunidades autónomas.

Este instrumento, al igual que otros disponibles en este ámbito y los que puedan desarrollarse en el futuro, no son sino herramientas al servicio de los y las profesionales. Ni este ni otros instrumentos podrán sustituir al juicio profesional; es más, su uso adecuado requiere de profesionales con formación y criterio. El instrumento orienta a los y las profesionales en la tarea de valorar la existencia de desprotección y su gravedad, les ayuda a focalizar su atención en las variables relevantes, y a hacerlo con criterios sólidos y consensuados. Pero cada niño, niña o adolescente y cada familia requieren una evaluación individualizada, centrada en sus circunstancias particulares. $Y$ la casuística es inmensa. Esto supone que en la aplicación del instrumento deberá lograrse el necesario equilibrio entre flexibilidad y fidelidad a sus fundamentos conceptuales, componentes centrales y criterios técnicos.
El instrumento BALORA no es una herramienta sencilla en una primera aproximación. Como ya se ha señalado, requiere conocer y asimilar previamente sus fundamentos conceptuales. Solo entonces el o la profesional estará preparado para utilizarlo. Posteriormente el instrumento le ayudará a realizar los procesos de recogida y análisis de la información de forma focalizada, rigurosa y meticulosa. En la medida en que el uso del instrumento sea más frecuente, el dominio sobre él aumentará y su manejo será más ágil. La provisión de formación y apoyo técnico, particularmente en las fases iniciales de utilización del instrumento, será importante para favorecer este proceso y corregir expectativas incorrectas o inadecuadas hacia él.

El instrumento BALORA es una herramienta dinámica que deberá adaptarse a los cambios legislativos, organizativos, sociales y a los avances científico-técnicos que vayan produciéndose. Los procesos de revisión periódica de su contenido y la monitorización y apoyo técnico a su implantación serán garantía del logro de sus objetivos y posibilitará su mejora permanente. 


\section{Bibliografía referenciada}

ARRUABARRENA, I. y DE PAÚL, J. (2012): “Improving accuracy and consistency in child maltreatment severity assessment in child protection services in Spain: New set of criteria to help caseworkers in substantiation decisions", Children \& Youth Services Review, vol. 34, n. $\stackrel{0}{-}$ 4, págs. 666-674. [<https://doi.org/10.1016/j. childyouth.2011.12.011)].

- (2011): "Valoración de la gravedad de las situaciones de desprotección infantil por los profesionales de los Servicios de Protección Infantil", Psicothema, vol. 23, n. 4 , págs. 642-647.

ARRUABARRENA, I.; DE PAÚL, J.; INDIAS, S.; y ULLATE, M. (2013): "Los profesionales de la psicología en la evaluación de la severidad de las situaciones de maltrato psicológico infantil en la familia", Psicothema, vol. 25, nํㅜ 4, pág. 482-487 [rhttps://doi.org/10.7334/ psicothema2013.146>].

CHILDREN`S BUREAU OF SOUTHERN CALIFORNIA (1997): Family Assessment Form. A practice- based approach to assessing family functioning, Washington, Child Welfare League of America.

DIPUTACIÓN FORAL DE ÁLAVA (2004): Guía de actuación para los Servicios Sociales dirigidos a la infancia en el Territorio Histórico de Álava, Vitoria-Gasteiz, Diputación Foral de Álava [<http://www.araba. eus/cs/Satellite?c=IFBS_Generico_FA\&cid=1 223988017985 \& pageid $=500002131563 \&$ pag ename=IFBS\%2FIFBS_Generico_FA\%2FIFBS generico〉].

DIPUTACIÓN FORAL DE BIZKAIA (2005): Intervención en situaciones de desprotección infantil, Bilbao, Diputación Foral de Bizkaia [<http://www. bizkaia.net/Home2/Archivos/DPTO3/Temas/ Pdf/MANUAL.pdf $>$ ].
DIPUTACIÓN FORAL DE GIPUZKOA (2003): Guía de actuación en situaciones de desprotección infantil. Recepción, investigación, evaluación inicial y elaboración del plan de intervención, DonostiaSan Sebastián, Diputación Foral de Gipuzkoa.

ENGLISH, D.J.; y THE LONGSCAN INVESTIGATORS (1997): Modified Maltreatment Classification System (MMCS) [<http://www.unc.edu/depts/sph/ longscan/pages/maltx/mmcs/LONGSCAN\%20 MMCS\%2oCoding.pdf $>$ ].

ESPAÑA (2015): “Ley 39/2015, de 1 de octubre, del Procedimiento Administrativo Común de las Administraciones Públicas", Boletín Oficial del Estado, n.ำ236, 2-10-2015, págs. 8934389410 [रhttps://www.boe.es/diario_boe/txt. php?id=BOE-A-2015-10565'].

- (2015): “Ley 26/2015, de 28 de julio, de modificación del sistema de protección a la infancia y a la adolescencia", Boletín Oficial del Estado, n. 0 180, 29-7-2015, págs. 64544-64613 [<https://www.boe.es/diario_boe/txt. php?id=BOE-A-2015-8470>].

- (2015): “Ley Orgánica 8/2015, de 22 de julio, de modificación del sistema de protección a la infancia y a la adolescencia”, Boletín Oficial del Estado, n. .0175, 23-7-2015, págs. 6187161889 ['https://www.boe.es/diario_boe/txt. php?id=BOE-A-2015-8222>].

MAGURA, S.; y MOSES, B.S. (1986): Outcome measures for child welfare services. Theory and applications, Washington, Child Welfare League of America.

ONTARIO ASSOCIATION OF CHILDREN'S AID SOCIETIES (2006): Eligibility Spectrum, Toronto, Ontario Association of Children's Aid Societies.

PAÍS VASCO (2017): “CORRECCIÓN DE ERRORES del Decreto $152 / 2017$, de 9 de mayo, por el que se aprueba 
la actualización del Instrumento para la valoración de la gravedad de las situaciones de riesgo y desamparo en los Servicios Sociales Municipales y Territoriales de Atención y Protección a la Infancia y adolescencia en la Comunidad Autónoma del País Vasco (BALORA)", Boletín Oficial del País Vasco, n.. 136, 18-7-2017 [khttps://www.euskadi.eus/ y22-bopv/es/bopv2/datos/2017/07/1703625a. shtml $]$.

- (2017): "DECRETO 152/2017, de 9 de mayo, por el que se aprueba la actualización del Instrumento para la valoración de la gravedad de las situaciones de riesgo y desamparo en los Servicios Sociales Municipales y Territoriales de Atención y Protección a la Infancia y adolescencia en la Comunidad Autónoma del País Vasco (Balora)", Boletín Oficial del País Vasco, n.ํㅜㄹ 3-7-2017 [rhttps://www.euskadi.eus/y22-bopv/es/ bopv2/datos/2017/07/1703354a.shtml>].

- (2015): “DECRETO 185/2015, de 6 de octubre, de cartera de prestaciones y servicios del Sistema Vasco de Servicios Sociales", Boletín Oficial del País Vasco, n.ำ206, 29-10-2015 [<https:// www.euskadi.eus/y22-bopv/es/bopv2/ datos/2015/10/1504561a.shtml>].

- (2011): “DECRETO 230/2011, de 8 de noviembre, por el que se aprueba el instrumento para la valoración de la gravedad de las situaciones de riesgo en los servicios sociales municipales y territoriales de atención y protección a la infancia y adolescencia en la Comunidad
Autónoma del País Vasco (BALORA)", Boletín Oficial del País Vasco, n.ํㅜ 233, 12-12-2011 [rhttps://www.euskadi.eus/y22-bopv/es/ bopv2/datos/2011/12/1105937a.shtmls].

- (2005): “Ley 3/2005, de 18 de febrero, de Atención y Protección a la Infancia y la Adolescencia", Boletín Oficial del País Vasco, n.ํ5, 59, 30-3-2005 [<https://www.euskadi.eus/y22-bopv/es/ bopv2/datos/2005/03/0501476a.shtmls].

SLEP, A.M.S.; y HEYMAN, R.E. (2004): “Severity of partner and child maltreatment: Reliability of scales used in American's largest child and family protection agency", Journal of Family Violence, vol. 19, n.ํㅜ 2, págs. 95-106 [<https://doi. org/10.1023/B:JOFV.0000019840.36496.a1)].

SRIVASTAVA, O.P.; y POLNAY, S. (1997): “Field trial of graded care profile (GCP) scale: A new measure of care", Archives of Disease in Childhood, vol. 76, págs. 337-340 [<http://dx.doi.org/10.1136/ adc.76.4.337〉].

STRAUS, M.A.; y HAMBY, S.L. (1997): “Measuring physical and psychological maltreatment of children with the Conflict Tactics Scale", en KANTOR, G.K.; y JASINSKI, J.L. (eds.), Out of the darkness: Contemporary perspectives on family violence, págs. 119-135, Thousand Oaks, Sage.

TROCMÉ, N. (1996): “Development and preliminary evaluation of the Ontario Child Neglect Index", Child Maltreatment, vol. 1, n.ํㅜ 2, págs. 145-155 [<https://doi.org/10.1177/1077559596001002 006)]. 